\title{
Minimum volume of long liquid bridges between noncoaxial, nonequal diameter circular disks under lateral acceleration
}

\author{
J. Meseguer, J. L. Espino, A. Cuerva, and A. Sanz-Andrés \\ IDR/UPM, E.T.S.I. Aeronáuticos, Universidad Politécnica de Madrid, E-28040 Madrid, Spain
}

(Received 1 July 2005; accepted 14 September 2005; published online 7 October 2005)

\begin{abstract}
The stability limit of minimum volume and the breaking dynamics of liquid bridges between nonequal, noncoaxial, circular supporting disks subject to a lateral acceleration were experimentally analyzed by working with liquid bridges of very small dimensions. Experimental results are compared with asymptotic theoretical predictions, with the agreement between experimental results and asymptotic ones being satisfactory. (C) 2005 American Institute of Physics.
\end{abstract}

[DOI: $10.1063 / 1.2107747]$

In the simplest configuration a liquid bridge consists of an isothermal drop of liquid held by surface tension forces between two parallel, solid disks as shown in Fig. 1. Disregarding electric and magnetic fields effects, the equilibrium interface shapes and hydrostatic stability limits of liquid bridges are determined by the slenderness, $\Lambda=L /\left(2 R_{0}\right)$, where $L$ is the distance between the supporting disks and the characteristic length $R_{0}$ is the mean radius, $R_{0}=\left(R_{1}+R_{2}\right) / 2$; the ratio of the radius of the smaller disk, $R_{1}$, to the radius of the larger one, $R_{2}$, that is $K=R_{1} / R_{2}$, or the equivalent parameter $h=(1-K) /(1+K)=\left(R_{2}-R_{1}\right) /\left(R_{2}+R_{1}\right)$; the dimensionless eccentricity, $e=E / R_{0}, 2 E$ being the distance between the disks axes; the dimensionless volume, defined as the ratio of the actual volume $\bar{V}$ to the volume of a cylinder of the same length $L$ and diameter $2 R_{0}: V=\bar{V} /\left(\pi R_{0}^{2} L\right)$; and the lateral Bond number, $B=\Delta \rho g R_{0}^{2} / \sigma$, where $\Delta \rho$ is the difference between the density of the liquid and the density of the surrounding medium, $g$ is the lateral acceleration acting on the liquid drop, and $\sigma$ is the surface tension.

The stability limit of the minimum volume of long axisymmetric liquid bridges held between unequal, noncoaxial parallel circular supporting disks subject to lateral acceleration can be theoretically analyzed by using an analytical approximation based on the standard bifurcation theory (Lyapunov-Schmidt technique $^{1}$ ). This problem was first analyzed a decade ago, both analytically and experimentally, ${ }^{2}$ with the following asymptotic expression for the stability limit of minimum volume obtained:

$$
\begin{aligned}
V_{\min }= & 1+\frac{2}{\pi} \Lambda+2\left(\frac{3}{2}\right)^{4 / 3}\left(\frac{1}{\pi} h+\frac{3}{2 \pi} B e \cos \beta\right)^{2 / 3} \\
& +\frac{\pi^{2}}{2} B^{2}+\frac{3}{2 \pi} e^{2},
\end{aligned}
$$

where the eccentricity $e$ is assumed to be positive when the relative position of the disks is as indicated in Fig. 1 (the smaller disk axis over the larger disk one). According to expression (1) the combined effect of lateral acceleration and eccentricity becomes maximum when the angle $\beta$ vanishes; this case is the only one considered in this Brief Communication. The variation of the minimum volume with the ec- centricity for a lateral Bond number $B=0.05$ and different values of the parameter $h$ (assuming $\beta=0$ ) of liquid bridges with slenderness $\Lambda=2.7$ is shown in Fig. 2 .

Expression (1) is only valid when the liquid bridge configuration is close enough to the reference one (defined by the Rayleigh stability limit, $\Lambda=\pi, V=1, B=h=e=0$ ), and far from this configuration of reference, Eq. (1) gives only a rough approximation of the influence on the stability limit of the different parameters considered. However, expression (1) shows the quantitative and the qualitative influence of the different parameters, which is determined by the exponent of each group of terms. Such an influence can be understood by considering the effect of the imposed perturbation on the necking of the liquid column. In fact, within the validity range of this asymptotic analysis the expression for both stable and unstable equilibrium interface shapes is

$$
f(z)=1+\varepsilon \sin \frac{\pi z}{\Lambda}
$$

where $\varepsilon$ is a small parameter that measures the magnitude of the interface deformation (whose value depends on the perturbations acting on the liquid bridge). Therefore, when the instability develops, the liquid bridge interface bulges in one half of the liquid column and necks in the other (the instability is antisymmetric with respect to the midplane parallel to the disks). According to this behavior, any perturbation leading to an antisymmetric deformation of the interface will decrease the maximum stable slenderness (that means to increase the minimum volume), the reduction being proportional to the two-thirds power of the perturbation. ${ }^{2}$ Note that since the difference in disk radii causes an antisymmetric deformation in the same manner as the combination of lateral Bond number and eccentricity (provided $\beta \neq \pi / 2$ ), these two effects can be additive or subtractive, and can even cancel one another.

The relative influence of the involved parameters $(h, B$, and $e$ ) on the instability can be studied by analyzing the size of the drops resulting after liquid bridge breakage. Since the unstable equilibrium interface shapes are given by expression (2), it is clear that any perturbation leading to an antisymmetric deformation of the interface will force a neck in 


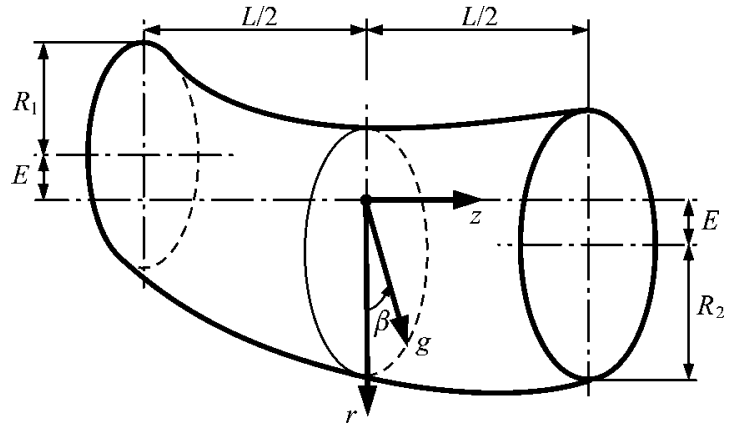

FIG. 1. Geometry and coordinate system for the liquid bridge problem.

the liquid bridge that will determine further the breaking process. When two antisymmetric effects act in opposite directions, the interface deformations associated to each one of the parameters will be also opposite, so that the position of the interface neck along the liquid bridge axis will depend on the relative importance of the parameters involved.

The neck migration is very accentuated when the fluid configuration is close to the peak corresponding to a local minimum of each minimum-volume stability limit curve (Fig. 2), which is reached at the critical value $e_{\text {crit }}=-2 h /(3 B)$. Actually, according to the quoted asymptotic analysis $^{2}$ the bifurcation equation $(\beta=0)$ is

$$
\lambda=\frac{1}{\varepsilon}\left(\frac{1}{\pi} h+\frac{3}{2 \pi} B e\right)-\frac{1}{2} v+\frac{\pi^{2}}{4} B^{2}+\frac{3}{4 \pi} e^{2}+\frac{3}{4} \varepsilon^{2},
$$

with $\lambda=1-\Lambda / \pi$ and $v=V-1$. Then, for each liquid bridge configuration $(h, B, e$, and $v)$ the stability limit is defined by the value $\lambda_{\min }$, where $\mathrm{d} \lambda / \mathrm{d} \varepsilon=0$. As is known, the bifurcation equation (3) has three roots for $\lambda>\lambda_{\min }$ (that means $\Lambda$ $<\Lambda_{\max }$ ), two of them representing unstable solutions whereas the third (the root with the smallest $|\varepsilon|$ ) corresponds to the stable solution. On the other hand, for $\lambda<\lambda_{\min }$ there is only one real root that represents an unstable configuration.

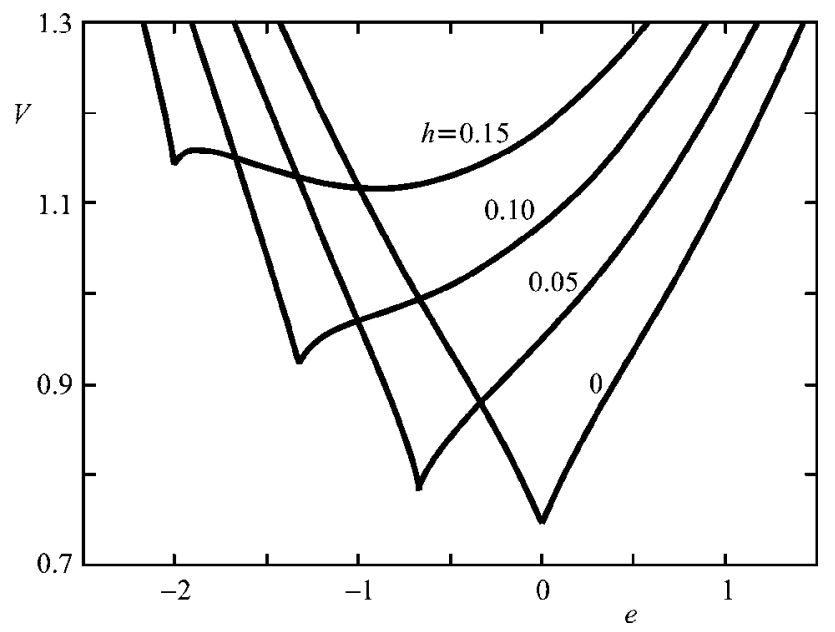

FIG. 2. Dimensionless minimum volume of the liquid bridge, $V$, vs eccentricity of the disks, $e$, for liquid bridges with slenderness $\Lambda=2.7$ under a lateral Bond number $B=0.05$ aligned with the plane defined by the disk axes $(\beta=0)$. Numbers on the curves indicate the value of the parameter $h$ that measures the ratio of the smaller disk diameter to the larger disk diameter.

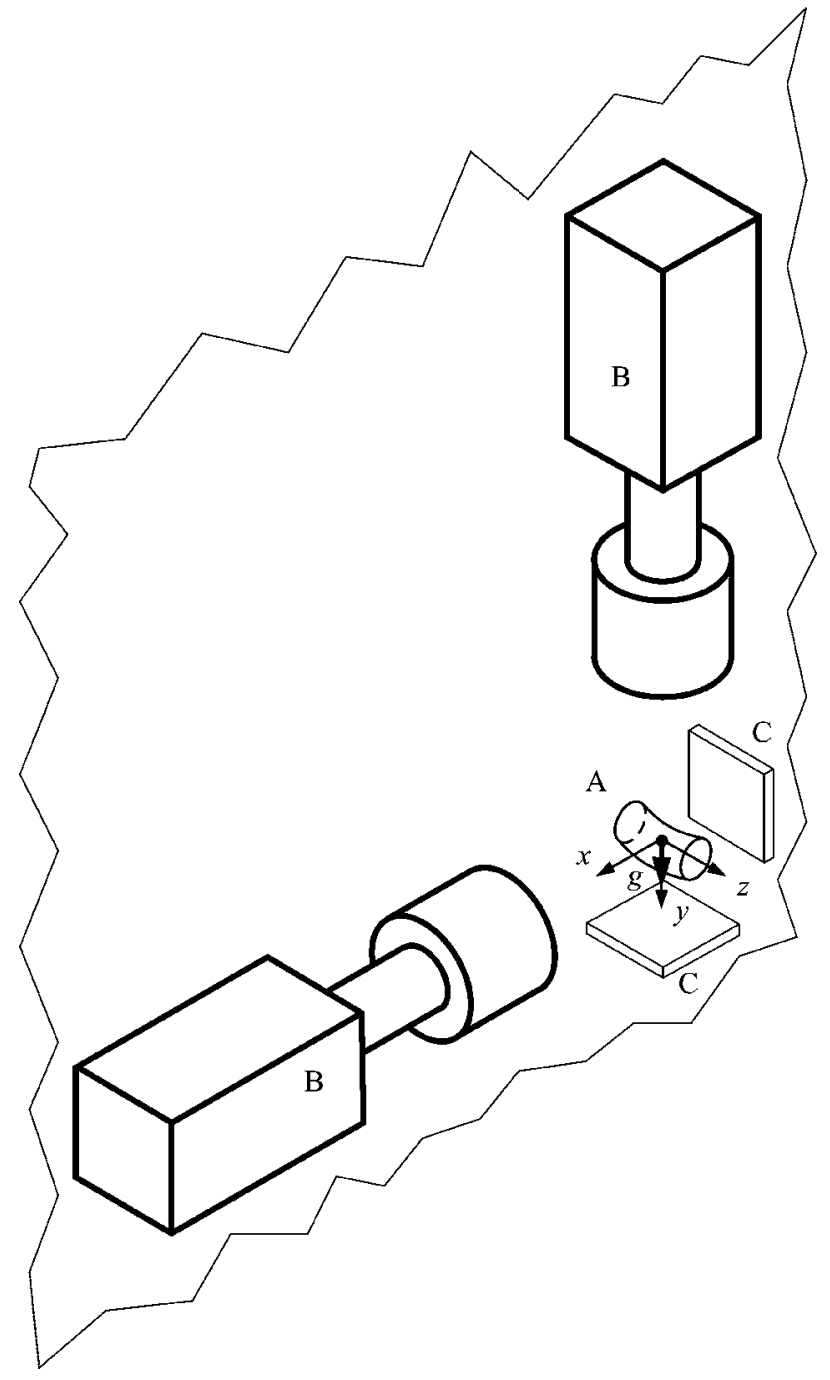

FIG. 3. Experimental apparatus: (A) liquid bridge cell, (B) CCD cameras, and $(\mathrm{C})$ background illumination devices.

Hence $\mathrm{d} \lambda / \mathrm{d} \varepsilon=0$ implies $\varepsilon=0$ if $2 h+3 B e=0$, or $\varepsilon$ $=[(2 h+3 B e) /(3 \pi)]^{1 / 3}$ in the case $2 h+3 B e \neq 0$. This last solution means that at the stability limit, irrespective of the value of the reduced slenderness, it will be $\varepsilon<0$ when $e$ $<e_{\text {crit }}=-2 h /(3 B)$. Therefore the liquid bridge will bulge close to the small disk and then a large drop will be formed at the smaller disk, and a small drop at the larger one after the liquid bridge rupture. On the other hand, when $e>e_{\text {crit }}$ it will be $\varepsilon>0$, hence the liquid bridge will neck in opposition, so that the size of the drops resulting after breaking will be just in opposition to the ones resulting when $e<e_{\text {crit }}$.

Aiming to experimentally check the above conclusion, an experimental apparatus like the one sketched in Fig. 3 has been used. The experimental setup consists of a vertical platform that supports the liquid bridge cell (A), two CCD cameras (B), and two LED background illumination devices (C). The liquid bridge cell, already described elsewhere, ${ }^{2-5}$ is a three-axes system that allows the displacement along each one of the axes through micrometric screws. One of the supporting disks is anchored to the $x, y$ plane (which in this arrangement is vertical) whereas the second one is fixed to the $z$-axis displacement screw. 
TABLE I. Nominal configurations used in experiments: radius of the larger disk, $R_{2}$, radius of the smaller disk, $R_{1}$, mean radius, $R_{0}$, unequal disks parameter, $h$, and lateral Bond number, $B$. In both configurations the liquid bridge slenderness was $\Lambda=2.7$.

\begin{tabular}{cccccc}
\hline \hline Configuration & $2 R_{2}(\mathrm{~mm})$ & $2 R_{1}(\mathrm{~mm})$ & $2 R_{0}(\mathrm{~mm})$ & $h$ & $B$ \\
\hline A & 1.47 & 1.47 & 1.47 & 0.000 & 0.074 \\
B & 1.47 & 1.17 & 1.32 & 0.114 & 0.059 \\
\hline \hline
\end{tabular}

Liquid bridge supports (disks) are made of brass tubes, their surfaces in contact with the working liquid being carefully polished to provide very sharp edges. One of these tubes (the one anchored to the $z$-axis displacement screw) is used for feeding and removing liquid, whereas the hole on the second one is sealed to avoid undesirable and uncontrolled changes of the liquid bridge volume during experimentation. The disk diameters used in experiments are indicated in Table I, as well as the main dimensionless parameters of the tested configuration. To estimate the nominal value of the lateral Bond number, it has been assumed that density and surface tension are the nominal values for water, $\rho=1000 \mathrm{~kg} / \mathrm{m}^{3}$ and $\sigma=0.072 \mathrm{~N} / \mathrm{m}$, respectively. In all the experiments the separation between the disks, $L$, has been appropriate to get a slenderness $\Lambda=2.7$.

A typical experiment is as follows: once the value of the angle $\beta=0$ is set by rotating the platform, supporting disks are placed close enough and a small amount of water is injected through the injection disk until a small liquid bridge is formed. Then the disks are slowly separated by moving the feeding disk along the $z$ axis and the opposite disk along the $y$ axis (vertical) until the desired configuration is reached (normally in this process some additional amount of water must be injected to prevent liquid bridge breakage during manipulation). When the desired configuration (slenderness and eccentricity) is reached, manipulation ceases and the experiment runs alone: because of evaporation the liquid bridge volume continuously decreases and the liquid bridge breaks when the stability limit is reached. Such a volume reduction

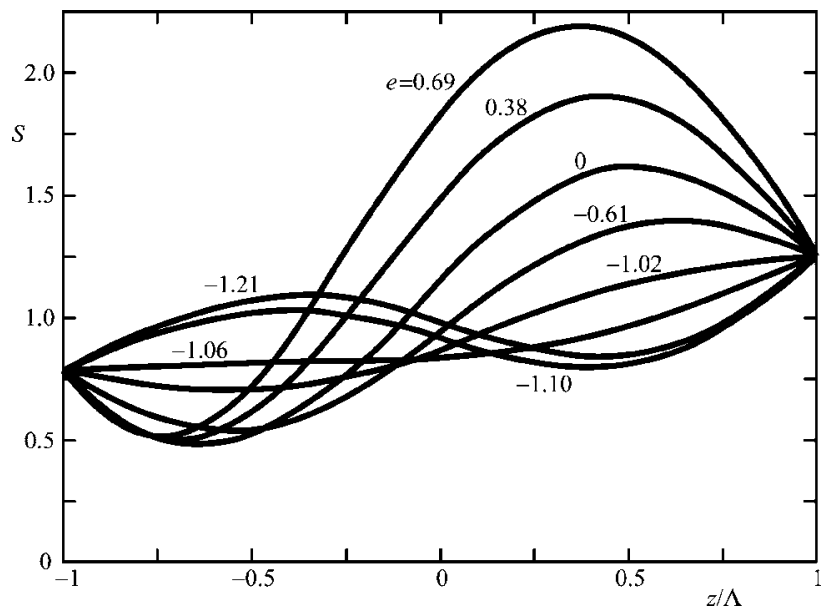

FIG. 4. Experimental results: Dimensionless cross-sectional area distributions, $S(z)$, of liquid bridges between unequal disks, $h=0.114$, subject to a lateral Bond number $B=0.059$. Numbers on the curves indicate the value of the eccentricity, $e$.
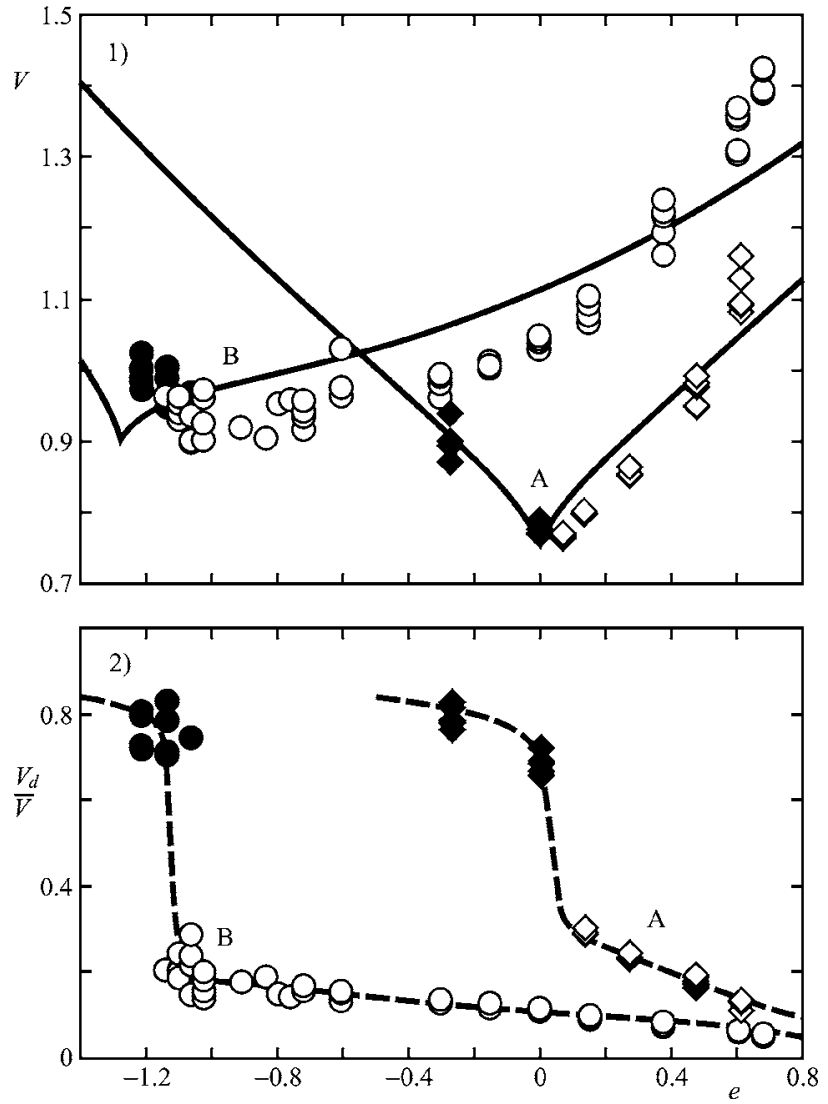

FIG. 5. Experimental results: (1) dimensionless minimum volume, $V$, vs eccentricity, $e$, and (2) relative volume of the liquid drop formed at the smaller disk, $V_{d} / V$, vs eccentricity, $e$, of liquid bridges between unequal disks. Labels identify the liquid bridge configuration as indicated in Table I. Symbols indicate the value of the relative volume of the liquid drop of reference: $V_{d} / V>0.5$ (solid symbols), $V_{d} / V<0.5$ (open symbols). Solid lines in (1) correspond to theoretical predictions according to expression (1), whereas dashed lines in (2) are only to show data trends.

process is recorded by the CCD cameras, so that from the recorded images just before the breaking, the liquid bridge contours are determined by using standard interface detection techniques already used in liquid bridge problems, ${ }^{6,7}$ and from these contours the liquid bridge volume, $V$ (the minimum volume stability limit), as well as the volume of liquid between the smaller disk and the liquid bridge neck, $V_{d}$, are calculated. To calculate such volumes it is assumed that liquid bridge cross sections are ellipses, which agrees with published analytical approximations for the shape of liquid bridge interfaces ${ }^{3,8}$ (additional details can be obtained upon request from the authors). Cross-sectional area distributions, $S(z)$, of liquid bridges at stability limits corresponding to different values of the eccentricity are represented in Fig. 4.

This process is repeated as many times as required to assure experimental results are representative (because of the large deformation of the liquid bridge in many experimental sequences, the liquid bridge interface spreads over the lateral surfaces of the supporting tubes, thus these sequences are rejected). Amongst all the valid experimental runs corresponding to a given value of the eccentricity, the crosssectional area distribution plotted in Fig. 4 corresponds to the 
run in which the smaller value of the minimum volume stability limit has been reached.

Experimental results corresponding to the configurations listed in Table I are shown in Fig. 5. As can be observed, experimental results corroborate the above theoretical predictions: at least when the parameters under consideration $(B$ and $h$ ) are small enough there is a critical value of the eccentricity and an associated minimum in the corresponding stability limit curve [Fig. 5(1)]. Obviously the agreement with asymptotic predictions is only qualitative because experimental configurations $(\Lambda=2.7)$ are far from the validity range of an asymptotic approach $(\Lambda \approx \pi)$.

It must be stressed that the relative volume $V_{d} / V$ changes drastically at $e=e_{\text {crit }}\left[e_{\text {crit }} \approx-2.2\right.$ for the configuration with $h=0.121$, Fig. 5(2)]. When $e<e_{\text {crit }}$ most of the liquid moves to the smaller disk when the liquid breaking occurs, while the larger drop appears at the larger disk when $e>e_{\text {crit }}$. Obviously, because of uncontrolled perturbations there is some scattering on the relative volume $V_{d}$ very close to the critical eccentricity, and the larger drop is formed either at the larger or at the smaller disk depending on the relative importance of the two effects under consideration (unequal disks and Bond number).
The authors are indebted to the referees for their helpful comments.

${ }^{1}$ N. S. Chow and J. K. Hale, Methods of Bifurcation Theory (Springer, Berlin, 1982).

${ }^{2}$ J. Meseguer, N. A. Bezdenejnykh, J. M. Perales, and P. Rodríguez de Francisco, "Theoretical and experimental analysis of stability limits of non-axisymmetric liquid bridges under microgravity conditions," Microgravity Sci. Technol. 8, 2 (1995).

${ }^{3}$ J. Meseguer, N. A. Bezdenejnykh, and P. Rodríguez de Francisco, "On the use of liquid bridges as accelerometers," Microgravity Sci. Technol. 9, 62 (1996).

${ }^{4}$ N. A. Bezdenejnykh, J. Meseguer, and J. M. Perales, "An experimental analysis of the instability of non-axisymmetric liquid bridges in a gravitational field," Phys. Fluids 11, 3181 (1999).

${ }^{5}$ J. Meseguer, J. L. Espino, J. M. Perales, and A. Laverón-Simavilla, “On the breaking of long, axisymmetric liquid bridges between unequal supporting disks at minimum volume stability limit," Eur. J. Mech. B/Fluids 22, 355 (2003).

${ }^{6}$ J. M. Perales and J. Meseguer, "Theoretical and experimental study of the vibration of axisymmetric viscous liquid bridges," Phys. Fluids A 4, 1110 (1992).

${ }^{7}$ G. Cabezas, J. M. Montanero, J. Acero, M. A. Jaramillo, and J. A. Fernández, "Detection of liquid bridge contours and its applications," Meas. Sci. Technol. 13, 829 (2002).

${ }^{8}$ J. M. Montanero, G. Cabezas, J. Acero, and J. M. Perales, "Theoretical and experimental analysis of the equilibrium contours of liquid bridges of arbitrary shape," Phys. Fluids 14, 682 (2002). 
Physics of Fluids is copyrighted by the American Institute of Physics (AIP). Redistribution of journal material is subject to the AIP online journal license and/or AIP copyright. For more information, see http://ojps.aip.org/phf/phfcr.jsp 